\title{
Plano amostral em parcelas de milho para avaliação de atributos de espigas
}

\author{
Sampling plan in corn plots to evaluate ear characteristics
}

\author{
Thomas Newton Martin ${ }^{1}$ Lindolfo Storck $^{2}$ Alessandro Dal’Col Lúcio ${ }^{3}$ \\ Leandro Homrich Lorent ${ }^{4}$
}

RESUMO

Em experimentos agrícolas, a amostragem é importante para reduzir os custos e a mão-de-obra, embora devam possuir características que confiram precisão experimental adequada. $O$ objetivo do trabalho foi determinar uma metodologia para se amostrar parcelas $e$ sub-parcelas experimentais de milho, quando o objetivo é avaliar atributos morfológicos e de rendimento avaliados nas espigas. $O$ experimento foi realizado, no Departamento de Fitotecnia da Universidade Federal de Santa Maria, com quatro bases genéticas de milho (híbrido simples - DAS 9560; híbrido duplo - AG 303; híbrido triplo - AG 6018; variedade cultivada - BR 202). O delineamento usado foi blocos completos casualizados com quatro repetições. A parcela foi constituída de 96 sub-parcelas de um metro. Em cada parcela, amostraram-se as espigas de cinco subparcelas. Foram avaliados o comprimento, o diâmetro, o rendimento de grãos e a massa de cem grãos de cada espiga. Concluiu-se que o tamanho da amostra nas parcelas de milho está relacionado ao atributo a ser avaliado nas espigas. Existem interferências genéticas e ambientais sobre a magnitude da estimativa do tamanho da amostra de espigas de milho. Pode-se amostrar 24 espigas por parcela, em seis repetições por genótipo, para avaliar os atributos comprimento e largura de espigas, rendimento de grãos e massa de cem grãos, para obter uma semi-amplitude do intervalo de confiança abaixo de $10 \%$ da média.

Palavras-chave: Zea mays $L$., número de repetições, subparcelas, precisão experimental.

\begin{abstract}
In agricultural experiments good sampling techniques are important to reduce costs and labor, and guarantee adequate experimental precision. The objective of this research was to study a sampling methodology of experimental plots to evaluate morphologic characteristic and corn yield in maize spikes. Single (DAS 9560), double (AG 6018) and triple (AG 303) crosses corn hybrids and an open pollinated variety (BR 202) were evaluated. The experiment design was a randomized complete block with four replications and was conducted in the experimental area of the Crop Science Department of the Federal University of Santa Maria (UFSM), Brazil. The main plot was divided in 96 sub plots with one meter of length. In each plot, the spikes of five sub-plots were sampled and length, diameter, grain yield and the mass of one hundred grains of each spike was measured or weighed. It was concluded that the sample size within plots is related to the characteristic to be evaluated in the maize spikes and there are genetic and environment factors that interfere on estimation of sample size of maize spikes. A sample of 24 spikes, one for each split-plot, and six replications for each genotype, results in a considered appropriate precision (less than 10\% from the mean) for length and width of spikes, grain yield and mass of one hundred grains.
\end{abstract}

Key words: Zea mays L., number of replications, split-plot, experimental precision.

\footnotetext{
${ }^{1}$ Escola Superior de Agricultura "Luiz de Queiroz" (ESALQ), Universidade de São Paulo (USP), Piracicaba, SP, Brasil. E-mail: martin@esalq.usp.br

${ }^{2}$ Departamento de Fitotecnia, Centro de Ciências Rurais (CCR), Universidade Federal de Santa Maria (UFSM), 97105-900, Santa

Maria, RS, Brasil. E-mail: lindolfo@smail.ufsm.br. Autor para correspondência

${ }^{3}$ Departamento de Fitotecnia, CCR, UFSM, Santa Maria, RS, Brasil.

${ }^{4}$ Programa de Pós-graduação em Agronomia (PPGA), UFSM, Santa Maria, RS, Brasil.
} 


\section{INTRODUÇÃO}

A execução de experimentos é, muitas vezes limitada pelo grande número de mensurações obtidas em cada unidade experimental. A amostragem é uma alternativa, porém ao dimensionar uma amostra aleatória simples, necessita-se do conhecimento prévio da variância da população e do grau de precisão desejado. Quando não se dispõem de informações sobre a variabilidade da população a ser amostrada, deve-se realizar uma pré-amostragem, em pequena escala, a fim de que se possa obter estimativas dos parâmetros populacionais (média e variância), que serão usados na obtenção do melhor tamanho da amostra (SILVEIRAJÚNIOR et al., 1980).

Segundo STEEL et al. (1997), para populações finitas existem três alternativas básicas de execução de plano amostral: (i) amostragem aleatória, (ii) amostragem sistemática e (iii) amostragem arbitrária. Assim, a diferença entre o valor estimado na amostra e o valor do parâmetro na população denomina-se erro amostral ou de amostragem (GOMEZ \& GOMEZ, 1984), e obviamente o erro de amostragem diminui à medida que o tamanho da amostra aumenta.

Estimativas do tamanho de amostra para avaliação do peso de espiga despalhada de famílias de meio-irmãos de milho, indicam que o maior número de plantas avaliadas proporciona resultados mais precisos (PALOMINO et al., 2000). Além disso, parcelas com o mesmo número de plantas, mas distribuídas em duas ou três linhas proporcionam maior precisão experimental e o ganho esperado com a seleção decresce com o aumento do número de plantas utilizadas por parcela.

$\mathrm{Na}$ avaliação do tamanho da amostra (número de espigas) nas parcelas de milho, para o percentual de umidade dos grãos, HENRY et al. (1942) verificaram que essa variou de três espigas (10 variedades cultivadas em cinco repetições) até 20 espigas (uma variedade cultivada sem repetição). Para peso de 50 grãos, peso de espiga e altura de planta, DIAS (1978) conclui que o tamanho da amostra deve ser de 13,15 e 5 plantas, respectivamente.

Métodos de amostragem foram pesquisados por SILVA \& SOUSA (1991), CONCEIÇÃO et al. (1993) e SILVA et al. (1993). Estes autores produziram informações sobre diferentes métodos de amostragem aleatória, para a variável altura de plantas e de inserção de espigas e do número de ramificações do pendão do milho. Além desses autores, SILVA et al. (1998) verificaram o efeito da amostragem aleatória e não aleatória sobre caracteres de milho.
O objetivo do trabalho foi determinar uma metodologia para se amostrar parcelas e sub-parcelas experimentais de milho, quando o objetivo é avaliar atributos morfológicos e de rendimento avaliados nas espigas. O melhor plano amostral será definido como aquele que propicia a menor estimativa da variância de médias de tratamentos.

\section{MATERIAL E MÉTODOS}

O experimento foi realizado no ano agrícola 2001/2002, no campus da Universidade Federal de Santa Maria, em solo pertencente à unidade de mapeamento Santa Maria, Brunizem Hidromórfico (EMBRAPA, 1999). Os tratamentos foram compostos de quatro bases genéticas de milho, sendo elas, híbrido simples (DAS 9560), híbrido duplo (AG 303), híbrido triplo (AG 6018) e variedade cultivada (BR 202). O manejo da cultura do milho foi de acordo com as indicações técnicas para a cultura de milho no estado do Rio Grande do Sul (INDICAÇÕES, 2001). O experimento foi implantado segundo o delineamento blocos completos casualizados com quatro repetições, usando parcelas de oito filas de $12 \mathrm{~m}$ de comprimento com $0,8 \mathrm{~m}$ entre filas. Cada parcela foi dividida em 96 sub-parcelas de um metro linear. Cinco das subparcelas, tomadas ao acaso, foram usadas para se avaliar o comprimento e o diâmetro $(\mathrm{cm})$ de todas as espigas, o rendimento de grãos (g/espiga) e a massa de cem grãos (g).

Os dados foram analisados segundo o modelo: $Y_{i j k l}=m+b_{j}+g_{i}+\varepsilon_{i j}+\tau_{i j k}+\delta_{i j k l}$, onde: $Y_{i j k l}$ é o valor observado referente a espiga $1\left(\mathrm{l}=1,2, \ldots, \mathrm{n}_{\mathrm{ijk}}\right.$, variando entre 1 e 5 espigas) da sub-parcela $\mathrm{k}(\mathrm{k}=1,2, \ldots, \mathrm{K}=5)$ do genótipo i $(\mathrm{i}=1,2, \ldots, \mathrm{I}=4)$ no bloco $\mathrm{j}(\mathrm{j}=1,2, \ldots, \mathrm{J}=4)$; $\mathrm{m}$ é a média; $b_{j}$ é o efeito aleatório do bloco $\mathrm{j}$; $\mathrm{g}_{\mathrm{i}}$ é o efeito fixo do genótipo i; $\varepsilon_{i j}$ é o efeito aleatório do erro experimental; $\tau_{i j k}$ é o efeito aleatório do erro entre as sub-parcelas; $\delta_{i j k l}$ é o efeito aleatório do erro entre espigas. Com as pressuposições usuais de normalidade com média igual a zero e independência para os

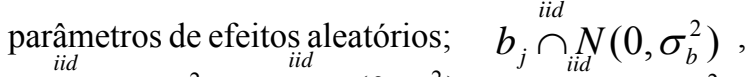
$\varepsilon_{i j} \cap N\left(0, \sigma_{\varepsilon}^{2}\right), \tau_{i j k} \cap N\left(0, \sigma_{\tau}^{2}\right)$ e $\delta_{i j k l} \cap N\left(0, \sigma_{\delta}^{2}\right)$. O quadro da análise da variância e as esperanças matemáticas dos quadrados médios estão representados na tabela 1 .

Usando a metodologia de estimação de componentes de variância (VENCOVSKY \& BARRIGA, 1992), foram calculadas: estimativa da variância entre espigas $\left(\hat{\sigma}_{\delta}^{2}=\mathrm{QM}_{\delta}\right)$; estimativa da variância entre subparcelas $\left(\hat{\sigma}_{\delta}^{2}=\left(\mathrm{QM}_{\tau}-\mathrm{QM}_{\delta}\right) / \mathrm{L}_{\mathrm{h}}\right)$ e estimativa da variância do erro experimental $\left(\hat{\sigma}_{\varepsilon}^{2}=\left(Q M_{\varepsilon}-Q M_{\tau}\right) / K L_{h}\right)$. Como 
Tabela 1 - Fontes de variação, graus de liberdade (GL), quadrados médios (QM) e esperança matemática dos QM.

\begin{tabular}{lccc}
\hline Fontes de variação & $\mathrm{GL}$ & $\mathrm{QM}$ & $\mathrm{E}(\mathrm{QM})$ \\
\hline Blocos & $\mathrm{J}-1$ & $\mathrm{QMb}$ & $\sigma_{\delta}^{2}+\mathrm{L}_{\mathrm{h}} \sigma_{\tau}^{2}+K \mathrm{~L}_{\mathrm{h}} \sigma_{\varepsilon}{ }^{2}+I K \mathrm{~L}_{\mathrm{h}} \sigma_{\mathrm{b}}^{2}$ \\
Genótipos & $\mathrm{I}-1$ & $\mathrm{QMg}$ & $\sigma_{\delta}^{2}+\mathrm{L}_{\mathrm{h}} \sigma_{\tau}^{2}+K \mathrm{~L}_{\mathrm{h}} \sigma_{\varepsilon}^{2}+J K \mathrm{~L}_{\mathrm{h}} \varphi(\mathrm{g})$ \\
Erro experimental & $(\mathrm{I}-1)(\mathrm{J}-1)$ & $\mathrm{QM} \varepsilon$ & $\sigma_{\delta}^{2}+\mathrm{L}_{\mathrm{h}} \sigma_{\tau}^{2}+K \mathrm{~L}_{h} \sigma_{\varepsilon}^{2}$ \\
Erro entre sub-parcelas & $\mathrm{IJ}(\mathrm{K}-1)$ & $\mathrm{QM} \tau$ & $\sigma_{\delta}^{2}+\mathrm{L}_{\mathrm{h}} \sigma_{\tau}^{2}$ \\
Erro entre espigas & $\sum_{i j k}\left(n_{i j k}-1\right)$ & $\mathrm{QM} \delta$ & $\sigma_{\delta}^{2}$ \\
\hline
\end{tabular}

* $n_{i j k}=$ número de espigas na sub-parcela $\mathrm{k}$ do genótipo i do bloco $\mathrm{j} ; \phi(g)=\sum_{i} g_{i}^{2} /(I-1)$

$\mathrm{n}_{\mathrm{ijk}}$ não é o mesmo para todas as sub-parcelas, usamos o valor médio estimado por

$$
L_{h}=\frac{1}{I J K-1}\left(n .-(1 / n .) \sum_{i j k} n_{i j k}^{2}\right)
$$

adaptado do delineamento inteiramente casualizado com diferente número de repetições (BARBIN, 1998).

Os coeficientes de variação, em percentagem, foram estimados para o erro experimental ( $C V_{\varepsilon}=100 \sqrt{Q M_{\varepsilon}} / \hat{m}$ ), para a o erro entre subparcelas $\left(C V_{\tau}=100 \sqrt{Q M_{\tau}} / \hat{m} \quad\right)$ e para o erro entre espigas $\left(C V_{\delta}=100 \sqrt{Q M_{\delta}} / \hat{m}\right)$.

A estimativa do tamanho da amostra (n) pode ser obtida pela expressão $\mathrm{n}=\mathrm{z}_{\alpha / 2}^{2} \sigma^{2} / \mathrm{d}^{2}$ em que d é a semi-amplitude do intervalo de confiança para a média, z é o valor crítico da distribuição normal padrão e $\sigma^{2}$ é a variância populacional (FONSECA \& MARTINS, 1994). Como, no caso, tem-se uma estimativa da variância $\left(\mathrm{s}^{2}\right)$ usa-se o valor crítico da distribuição t. Para generalizar a expressão de n, substituí-se $D=100 d / \bar{X}$ e $C V=100 \sqrt{s^{2}} / \bar{X}$ na expressão de $n$ e obtém-se $n=t^{2}{ }_{\alpha / 2} \mathrm{CV}^{2} / \mathrm{D}^{2}$, em que o $\mathrm{CV}$ é o coeficiente de variação (em percentagem), D é a semi-amplitude do intervalo de confiança (em percentagem) para a média (estabeleceu-se $\mathrm{D}=10 \% \mathrm{e}$ $\mathrm{D}=20 \%$ ) e té o valor crítico da distribuição t, em nível de $5 \%$ de probabilidade de erro.

$$
\text { Como } \hat{V} \hat{m}_{i}=\frac{Q M_{\varepsilon}}{J K L_{h}}=\frac{\hat{\sigma}_{\delta}^{2}}{J K L_{h}}+\frac{\hat{\sigma}_{\tau}^{2}}{J K}+\frac{\hat{\sigma}_{\varepsilon}^{2}}{J}
$$

é a estimativa da variância da média estimada para o genótipo i, dependendo das significâncias das variâncias $\left(\sigma_{\varepsilon}^{2}, \sigma_{\tau}^{2}\right.$ e $\left.\sigma_{\delta}^{2}\right)$ pode-se identificar a combinação $\mathrm{J}, \mathrm{Ke} \mathrm{L} \mathrm{L}_{\mathrm{h}}$ que minimiza a estimativa $\hat{V}\left(\hat{m}_{i}\right)$.

Nestas combinações, usa-se as restrições de valores mínimos $\mathrm{J}=2, \mathrm{~K}=1$ e $\mathrm{L}_{\mathrm{h}}=1$, mantendo-se fixa a quantia $\mathrm{JKL}_{\mathrm{h}}=4 * 5 * 5=100$ espigas por genótipo.

Devido ao pressuposto de efeito aleatório de blocos, na estimativa $\hat{V}\left(\hat{m}_{i}\right)$ deveria ser somada a componente de variância de blocos. No entanto, como nos testes de comparação múltipla de médias de Tukey utiliza-se a metade de $\hat{V}\left(\hat{m}_{i}-\hat{m}_{i^{\prime}}\right)$, cuja estimativa independe do bloco ser de efeito fixo ou aleatório, conforme mostrado para o delineamento blocos completos casualizados (STORCK \& LOPES, 1998), procedemos $\hat{V}\left(\hat{m}_{i}\right)=\hat{V}\left(\hat{m}_{i}-\hat{m}_{i^{\prime}}\right) / 2$ para fins de facilitar a obtenção da combinação $\mathrm{J}, \mathrm{K} \mathrm{e} \mathrm{L}_{\mathrm{h}}$ que minimiza $\hat{V}\left(\hat{m}_{i}\right)$.

\section{RESULTADOS E DISCUSSÃO}

O valor médio do número de espigas nas 80 sub-parcelas (16 parcelas x cinco sub-parcelas) amostradas foi $\mathrm{L}_{h}=3,18$. Comprimento e diâmetro de espigas não tiveram efeitos significativos para o erro entre sub-parcelas e para o erro experimental, sendo suas estimativas baixas ou nulas (Tabela 2). Neste caso, por exemplo, para comprimento de espiga, a estimativa da variância da média estimada de um genótipo é $\hat{V}\left(\hat{m}_{i}\right)=\frac{7,72}{J K L_{h}}+\frac{0}{J K}+\frac{0,11}{J} \cong \frac{7,72}{J K L_{h}}$, devido a nãosignificância de $\sigma_{\tau}^{2}$ e $\sigma_{\varepsilon}^{2}$. Valores nulos para os componentes de variâncias são admitidos quando a estimativa é um valor negativo. Ao se constatar problemas dessa natureza, pode-se admitir que o valor do parâmetro seja zero e sua estimativa como nula (SEARLE, 1971; BARBIN, 2003).

Como o número de repetições $(\mathrm{J})$ e o número de sub-parcelas (K) são denominadores das 
Tabela 2 - Fontes de variação, graus de liberdade (GL), estimativas dos quadrados médios para comprimento (CE), diâmetro (DE), rendimento de grãos $(\mathrm{RG})$ e massa de cem grãos $(\mathrm{MCG})$ de espiga de milho, estimativa da variância do erro experimental $\left(\hat{\sigma}_{e}^{2}\right)$, da variância entre as sub-parcelas $\left(\hat{\sigma}_{\tau}^{2}\right)$ e da variância entre espigas $\left(\hat{\sigma}_{\delta}^{2}\right)$ e respectivos coeficientes de variação em percentagem $(\mathrm{CV})$.

\begin{tabular}{llllll}
\hline \multicolumn{1}{c}{ Fontes de variação } & $\mathrm{GL}$ & $\mathrm{CE}(\mathrm{cm})$ & $\mathrm{DE}(\mathrm{cm})$ & $\mathrm{RG}(\mathrm{g})$ & $\mathrm{MCG}(\mathrm{g})$ \\
\hline Blocos & 3 & $29,98^{*}$ & $1,49^{*}$ & $26810,8^{*}$ & $17,70^{\mathrm{ns}}$ \\
Genótipos & 3 & $12,47^{*}$ & $0,95^{\mathrm{ns}}$ & $8997,3^{\text {ns }}$ & $182,35^{*}$ \\
Erro experimental & 9 & $9,15^{\text {ns }}$ & $0,33^{\text {ns }}$ & $6161,9^{*}$ & $16,58^{\text {ns }}$ \\
Erro entre sub-parcelas & 64 & $7,25^{\text {ns }}$ & $0,19^{\text {ns }}$ & $2218,7^{\text {ns }}$ & $28,42^{*}$ \\
Erro entre espigas & 209 & 7,72 & 0,19 & 2215,6 & 15,01 \\
Média & - & 14,67 & 4,27 & 134,59 & 32,85 \\
$\hat{\sigma}_{\varepsilon}^{2}\left(\mathrm{CV}_{\varepsilon}\right)$ & - & $0,11(20,6)$ & $0,01(13,5)$ & $246,5(58,3)$ & $0,00(12,4)$ \\
$\hat{\sigma}_{\tau}^{2}\left(\mathrm{CV}_{\tau}\right)$ & - & $0,00(18,4)$ & $0,00(10,2)$ & $0,9(35,0)$ & $4,19(16,2)$ \\
$\hat{\sigma}_{\delta}^{2}\left(\mathrm{CV}_{\delta}\right)$ & - & $7,72(18,9)$ & $0,19(10,2)$ & $2215,6(35,0)$ & $15,01(11,8)$ \\
\hline
\end{tabular}

* Significativo pelo teste $\mathrm{F}$ em nível de 5\% de probabilidade de erro; ns não-significativo.

estimativas não significativas de $\sigma_{\varepsilon}^{2}$ e $\sigma_{\tau}^{2}$, pode-se $\operatorname{minimizar} \hat{V}\left(\hat{m}_{i}\right)$ usando menores valores de $\mathrm{J}$ e K (por exemplo, $\mathrm{J}=2 \mathrm{e} \mathrm{K}=1$ ) em favor de um maior número de espigas por sub-parcela $\left(\right.$ maior $\mathrm{L}_{\mathrm{h}}$ ). Nestes atributos, não há vantagem em se agrupar espigas em subparcelas ou usar maior número de repetições. Deve-se estimar o número de espigas $\left(\mathrm{L}_{\mathrm{h}}\right)$ por parcela para cada genótipo conforme a expressão $\mathrm{n}=\mathrm{t}_{\alpha / 2}^{2} \mathrm{CV}^{2} / \mathrm{D}^{2}$ Assim, o tamanho da amostra para estimar o comprimento de espiga, para uma semi-amplitude do intervalo de confiança para a média de genótipo (D) igual a $10 \%$, foi igual a 19 espigas e para $\mathrm{D}=20 \%$ foi igual a sete espigas, para $5 \%$ de probabilidade de erro. A interpretação para o atributo diâmetro de espiga é semelhante, sendo a estimativa do tamanho da amostra igual a nove e quatro espigas, respectivamente, para $\mathrm{D}=10 \%$ e $\mathrm{D}=20 \%$, em nível de $5 \%$ de probabilidade de erro. Entretanto, como não é prático amostrar número diferente de espigas para determinar o comprimento e o diâmetro das espigas, pode-se adotar o máximo de 19 espigas para $\mathrm{D}=10 \%$ ou sete espigas para $\mathrm{D}=20 \%$. No caso de se usar $\mathrm{J}=2$ repetições, pode-se amostrar 10 espigas por repetição em cada genótipo, pois $n=\mathrm{JL}_{\mathrm{h}}$, aproximadamente igual a 20 .

Para o rendimento de grãos das espigas, a interação genótipo x bloco (erro experimental) foi significativa e o erro entre sub-parcelas não foi significativo (Tabela 2). Assim, a variação entre repetições não é a mesma nos diferentes genótipos, possivelmente devido à variação significativa entre blocos. Neste caso, a estimativa da variância do erro experimental referente a cada genótipo (decomposição do erro) pode ser obtida, aproximadamente, pela expressão $\operatorname{Var}\left(g_{i}\right)=\sum_{j} \hat{\varepsilon}_{i j}^{2} /(J-1)$ e observada na tabela 3. Nesta tabela, a relação entre a maior variância (genótipo AG 6018) e a menor variância (genótipo DAS 9560) é igual a 3,8, caracterizando a heterogeneidade das variâncias em relação aos genótipos. Por isto, deve-se estimar o tamanho da amostra separadamente para cada genótipo.

Ainda, para o atributo rendimento de grãos, a estimativa $\hat{V}\left(\hat{m}_{i}\right)=\frac{2215,6}{J K L_{h}}+\frac{0,9}{J K}+\frac{246,5}{J}$ indica que o número de repetições (J) é mais importante para se reduzir $\hat{V}\left(\hat{m}_{i}\right)$ e o tamanho da amostra independe do agrupamento das espigas em sub-parcelas, pois $\sigma^{2}{ }_{\tau}$ não foi significativo. Assim, o tamanho da amostra é dependente do genótipo e, por isto, deve-se amostrar cada genótipo com número específico de espigas ou usar o maior número de espigas, contemplando todos os genótipos com a precisão estipulada. Neste caso, para uma precisão $\mathrm{D}=10 \%$, o uso de $\mathrm{n}=148$ espigas é mais prático e, como $\mathrm{n}=\mathrm{JL}_{\mathrm{h}}=148$ (adotamos $\mathrm{K}=1$ ) e sendo $\mathrm{J}$ mais importante, pode-se reorganizar o delineamento para o máximo número de repetições. Por exemplo, ao usar $\mathrm{J}=6$ repetições deve-se amostrar 24 espigas por parcela.

Problemas com sensibilidade ambiental diferente entre genótipos de milho (NASPOLINI FILHO, 1975; SILVA et al., 1998) e outras culturas (ESTEFANEL et al., 1996) causam variação da heterogeneidade de variâncias. Estudo realizado por 
NASPOLINI FILHO (1975), confirma a teoria de que a variância é crescente no sentido do híbrido simples para os híbridos triplo, duplo e variedade cultivada. No presente estudo, a ordem crescente das variâncias (Tabela 3) não foi exatamente a mesma, talvez porque os genótipos usados não são representantes típicos de cada tipo de híbrido. O importante é observar que existem diferenças a serem consideradas.

Para massa de cem grãos, verificou-se significância para o efeito do erro entre sub-parcelas e não-significância para o erro experimental (Tabela 2), por isto, a variância do erro entre sub-parcelas foi estimada, para cada parcela, pela expressão $\operatorname{Var}\left(\tau_{i j}\right)=\sum_{k} \hat{\tau}_{i j k}^{2} /(K-1)$ podendo ser observada na tabela 4 .

Tabela 3 - Estimativas da variância do erro experimental referente a cada genótipo (Var), média (g/espiga), coeficiente de variação (CV\%) e número de espigas a serem amostrados em dois níveis de precisão ( $D=10$ e $20 \%$ da média) para a estimativa do rendimento de grãos de milho.

\begin{tabular}{lccccc}
\hline Genótipo & Var & Média & CV\% & D = 10\% & D = 20\% \\
\hline BR 202 & 2059,2 & 118,3 & 38,3 & 58 & 16 \\
AG 303 & 5520,0 & 129,8 & 57,2 & 128 & 34 \\
AG 6018 & 6987,2 & 135,2 & 61,8 & 148 & 39 \\
DAS 9560 & 1841,6 & 147,8 & 29,0 & 34 & 10 \\
\hline
\end{tabular}

O tamanho da amostra para uma precisão $\mathrm{D}=10$ e $20 \%$ (Tabela 4) se refere ao número de espigas em cada parcela ou seja, $n=K_{h}$. Pela expressão $\hat{V}\left(\hat{m}_{i}\right)=\frac{15,01}{J K L_{h}}+\frac{4,19}{J K}+\frac{0}{J} \quad$ observa-se que o aumento do número de repetições (J) não é eficiente para reduzir a $\hat{V}\left(\hat{m}_{i}\right)$. Maior eficiência pode ser obtida ao manter $\mathrm{L}_{\mathrm{h}}=1$ e maximizar $\mathrm{K}$ (número de sub-parcelas). Assim, o tamanho da amostra na parcela (n) deve ser obtida pela retirada de uma espiga em $n$ sub-parcelas.

Por exemplo, ao adotar $\mathrm{J}=4$ repetições, o número de sub-parcelas (espigas) a ser amostrada (Tabela 4) varia em função do genótipo e do bloco. Se optarmos pelo número máximo de espigas para $\mathrm{D}=10 \%$ (52 espigas no bloco dois para o genótipo BR 202) se estaria frente a um valor extremo e, se por outro lado, usar a média não se estaria propiciando a precisão desejada a todas as parcelas. Como a amplitude de variação do tamanho da amostra entre médias de bloco é menor do que a amplitude de variação entre média de genótipos, poder-se-ia usar a maior média do tamanho de amostra para genótipo (BR 202) que, neste caso será igual a 24 espigas por parcela (para $\mathrm{D}=10 \%$ ).

Para o conjunto dos quatro atributos avaliados nas espigas de milho, existem variações da forma de amostrar nas parcelas. Existem casos em que, ora a sub-parcela e ora o número de repetições é mais eficiente para aumentar a precisão experimental. Usando a situação da amostragem do número de

Tabela 4 - Estimativa da variância do erro entre sub-parcelas (Var) para cada parcela (Genótipo x bloco), médias, coeficiente de variação $(\mathrm{CV} \%)$ e número de espigas a serem amostrados em dois níveis de precisão $(\mathrm{D}=10$ e $20 \%$ da média) para a estimativa da massa de cem grãos de milho.

\begin{tabular}{|c|c|c|c|c|c|c|}
\hline Genótipo & Bloco & Var & Média (2) & CV\% & $\mathrm{D}=10 \%$ & $\mathrm{D}=20 \%$ \\
\hline BR 202 & 1 & 19,25 & 35,61 & 12,32 & 8 & 4 \\
\hline AG 303 & 1 & 40,39 & 32,17 & 19,76 & 17 & 6 \\
\hline AG 6018 & 1 & 21,51 & 31,67 & 14,65 & 11 & 4 \\
\hline DAS 9560 & 1 & 5,02 & 31,13 & 7,20 & 5 & 3 \\
\hline BR 202 & 2 & 167,68 & 35,94 & 36,03 & 52 & 15 \\
\hline AG 303 & 2 & 19,10 & 33,84 & 12,91 & 9 & 4 \\
\hline AG 6018 & 2 & 2,95 & 31,84 & 5,39 & 4 & 3 \\
\hline DAS 9560 & 2 & 8,65 & 34,22 & 8,59 & 5 & 3 \\
\hline BR 202 & 3 & 46,97 & 35,34 & 19,39 & 16 & 6 \\
\hline AG 303 & 3 & 19,19 & 33,08 & 13,24 & 9 & 4 \\
\hline AG 6018 & 3 & 7,91 & 32,88 & 8,55 & 5 & 3 \\
\hline DAS 9560 & 3 & 50,78 & 32,06 & 22,23 & 21 & 7 \\
\hline BR 202 & 4 & 57,60 & 36,76 & 20,65 & 19 & 7 \\
\hline AG 303 & 4 & 13,39 & 32,55 & 11,24 & 7 & 4 \\
\hline AG 6018 & 4 & 13,45 & 30,69 & 11,95 & 8 & 4 \\
\hline DAS 9560 & 4 & 1,09 & 31,17 & 3,34 & 3 & 3 \\
\hline
\end{tabular}


espigas para rendimento de grãos por espiga, a opção por amostrar 24 espigas por parcela em seis repetições por genótipo leva a uma precisão suficiente para os demais atributos. Além disso, é mais conveniente amostrar uma espiga em cada uma das 24 sub-parcelas compostas por até cinco plantas. Esta conclusão concorda com a encontrada por SILVA et al. (1998), quando recomendou 11 espigas para as características número de grãos por espiga, comprimento e peso do sabugo.

\section{CONCLUSÃO}

O tamanho da amostra nas parcelas de milho está relacionado ao atributo a ser avaliado nas espigas. Existem interferências genéticas e ambientais sobre a magnitude da estimativa do tamanho da amostra de espigas de milho. Pode-se amostrar 24 espigas por parcela, em seis repetições por genótipo, para avaliar os atributos comprimento e largura de espigas, rendimento de grãos e massa de cem grãos, para obter uma semi-amplitude do intervalo de confiança abaixo de $10 \%$ da média.

\section{REFERÊNCIAS}

BARBIN, D. Componentes de variância: teoria e aplicações. 2.ed. Piracicaba: FEALQ, 1998. 120p.

BARBIN, D. Planejamento e análise estatística de experimentos agronômicos. Arapongas: Midas, 2003. 208p.

CONCEIÇÃO, M.M. et al. Efeitos de bordadura e amostragem em experimentos com milho. Ciência Agronômica, v.24, p.63-69, 1993.

DIAS, J.F.S. O tamanho da amostra para estudo dos caracteres peso de 50 grãos, peso de espiga e altura da planta em quatro grupos de cultivares de milho (Zea mays, L.). 1978. 97f. Dissertação (Mestrado em Agronomia) - Programa de Pós-graduação em Genética e Melhoramento de Plantas, Universidade de São Paulo.

EMBRAPA. Centro Nacional de Pesquisa de Solos (Rio de Janeiro, RJ). Sistema brasileiro de classificação dos solos. Brasília: Embrapa-SPI, 1999. 412p.

ESTEFANEL, V. et al. Tamanho da amostra para avaliação de componentes do rendimento na cultura do feijoeiro. Ciência Rural, v.26, p.367-370, 1996.
FONSECA, J.S.; MARTINS, G.A. Curso de estatística. 5.ed. São Paulo: Atlas, 1994. 317p.

GOMEZ, K.A.; GOMEZ, A.A. Statistical procedures for agricultural research. 2.ed. New York: John Wiley, 1984. $680 \mathrm{p}$.

HENRY, G.F. et al. An adequate sample of corn plots with reference to moisture and shelling percentages. Agronomy Journal, n.34, p.777-781, 1942.

INDICAÇÕES técnicas para a cultura de milho no Estado do Rio Grande do Sul. Porto Alegre: FEPAGRO; EMBRAPA TRIGO, EMATER/RS; FECOAGRO/RS, n.7, 2001. 135p.

NASPOLINI FILHO, V. Variabilidade fenotípica e estabilidade em híbridos simples, híbridos duplos, variedades e compostos de milho (Zea mays L.). 1975. 68f. Dissertação (Mestrado em Agronomia) - Programa de Pós-graduação em Genética e Melhoramento de Plantas) Universidade de São Paulo.

PALOMINO, E.C. et al. Tamanho de amostra para avaliação de famílias de meios-irmãos de milho. Pesquisa Agropecuária Brasileira, v.35, p.1433-1439, 2000.

SEARLE, S.R. Linear models. New York: John Wiley \& Sons, 1971. 531p.

SILVA, J. et al. Amostragem e tamanho da amostra na estimação de caracteres da espiga do milho. Pesquisa Agropecuária Brasileira, v.33, p.19831988. 1998.

SILVA, P.S.L. et al. Método de amostragem e tamanho de amostra para alguns caracteres de milho. Ciência Agronômica, v.24, p.5-10, 1993.

SILVA, P.S.L.; SOUSA, R.P. Técnicas experimentais para o milho. Mossoró: Fundação Guimarães Duque/ESAM, 1991. 192 p.

SILVEIRA JÚNIOR, P. et al. Estatística geral - inferência estatística. Pelotas: UFPEL - DME, 1980. 156p.

STEEL, R.G.D. et al. Principles and procedures of statistics. 3.ed. Nova York: McGraw Hill Book, 1997. $666 \mathrm{p}$.

STORCK, L.; LOPES, S.J. Experimentação II. Santa Maria: UFSM - Departamento de Fitotecnia, 1998. 205p.

VENCOVSKY, R.; BARRIGA, P. Genética biométrica no fitomelhoramento. Ribeirão Petro: Revista Brasileira de Genética, 1992. 496p. 